\title{
The effect of aberrant maspin expression on the invasive ability of pancreatic ductal adenocarcinoma cells
}

\author{
SUNG NOH HONG ${ }^{1,2}$, JONG KYUN LEE ${ }^{3}$, WON HYOEK CHOE ${ }^{2}$, HYE YOUNG HA $^{4}$, KYOUNGSOOK PARK ${ }^{5}$, \\ IN KYUNG SUNG ${ }^{2}$, KYU TAEK LEE ${ }^{3}$, JAE J. KIM ${ }^{3}$ and JONG CHUL RHEE ${ }^{3}$ \\ ${ }^{1}$ Health Promotion Center, Konkuk University Hospital; ${ }^{2}$ Department of Internal Medicine, Konkuk University Hospital, \\ Konkuk University School of Medicine; ${ }^{3}$ Department of Medicine, Samsung Medical Center, Sungkyunkwan University \\ School of Medicine; ${ }^{4}$ Department of Laboratory Medicine, Konkuk University Hospital; \\ ${ }^{5}$ Molecular Therapy Research Center, Sungkyunkwan University, Seoul, Korea
}

Received August 28, 2008; Accepted November 20, 2008

DOI: $10.3892 /$ or_00000240

\begin{abstract}
Maspin is expressed aberrantly in pancreatic ductal adenocarcinoma (PDAC), and its function is still unknown. To explore the role of maspin in PDAC, we constructed wild-type maspin-expressing Panc-1 clones (Panc-1-maspin) by transfecting maspin cDNA into Panc-1, a PDAC-derived cell line that lacks maspin expression. As a control, mock-transfected clones (Panc-1-mock) were constructed using the same method. The invasive ability of the stable transfectants was evaluated with an in vitro invasion assay. The ability of Panc-1-maspin cells to migrate through a Matrigel-coated filter was significantly reduced compared to that of Panc-1-mock cells $(\mathrm{p}=0.012)$. In addition, we identified the c.1022A $>\mathrm{G}$ variant of maspin in human PDAC cells; however, this polymorphism was not involved in the clinical characteristics of PDAC nor did it alter the invasive ability of PDAC cells. The results of the present study indicate that maspin suppresses the invasive ability of PDAC cells.
\end{abstract}

\section{Introduction}

Maspin (mammary serine protease inhibitor, SERPINB5) is a member of the serine protease inhibitor family with tumor suppressing activity in breast cancer. Maspin expression was found in normal breast epithelial cells, but was frequently decreased in breast cancer cells and lost in metastatic cells $(1,2)$. Since the emerging studies on maspin demonstrated its ability to inhibit cell invasion, promote apoptosis, and inhibit angiogenesis, maspin has been classified as a class II tumor suppressor (3-6). In human breast carcinogenesis, the loss of

Correspondence to: Dr Jong Kyun Lee, Department of Medicine, Samsung Medical Center, Sungkyunkwan University School of Medicine, 50 Ilwon-dong, Kangnam-gu, Seoul 135-710, Korea E-mail: jongk.lee@samsung.com

Key words: maspin, pancreatic ductal adenocarcinoma, invasive ability, in vitro invasion assay maspin expression is classified as an early event (7) that is critical for cancer cell motility, invasion, and metastasis $(8,9)$.

The pattern of maspin expression in the pancreas, however, is at odds with that found in the breast. While normal pancreatic ductal epithelia have reduced or absent expression, maspin appears to be strongly expressed in most pancreatic ductal adenocarcinomas (PDAC) (10-12). Moreover, overexpression of maspin in PDAC is associated with an advanced tumor stage and poor postoperative survival $(13,14)$. Maspin expression in PDAC is aberrant when considering the discrepancy between the tumor suppressor properties of maspin and the clinical manifestations of PDAC. In general, PDAC is characterized by rapid local invasion of adjacent structures and early metastases in lymph nodes and the liver. The role of maspin in the aggressive phenotype of PDAC cells, however, has not been studied. Thus, we undertook the present study to evaluate the effect of maspin on the invasive ability of PDAC cells by transfecting maspin cDNA into PDAC cells that lack maspin.

Although no somatic mutations have been described for maspin, c.1022A>G (GenBank accession no. rs1455555) was found in the first codon position of amino acid 319 in some prostate cancer cell lines and in human prostate cancer tissues (15). Since this allelic variant results in an amino acid substitution of valine in place of isoleucine, this variant could be associated with the specific clinical features of a tumor and/ or the alteration of maspin's function. To investigate the significance of this allelic variant, we first identified this allelic variant in PDAC. We then searched for any association of this variant with the clinical characteristics of PDAC and the altered invasive ability of PDAC cells.

\section{Materials and methods}

Patients and clinical specimens. Human PDAC tissue specimens were obtained from a series of 40 patients (24 men and 16 women; average age, 56.0 \pm 9.4 years at diagnosis) who had undergone resection surgery for PDAC between 2000 and 2004 at Samsung Medical Center, Seoul, Korea. The surgically resected specimens of PDAC were fixed in $10 \%$ formalin and routinely processed for paraffin embedding. 

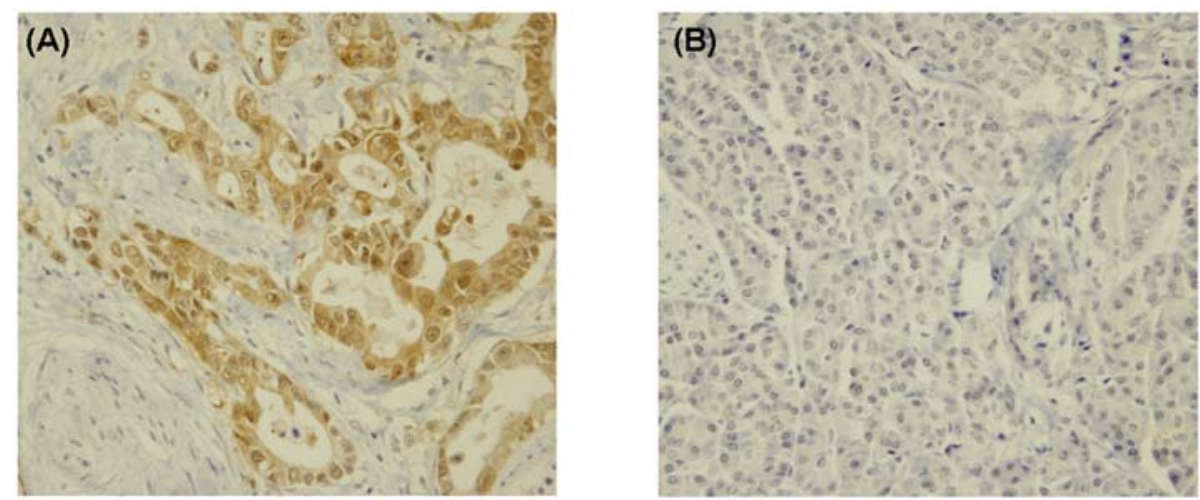

Figure 1. Immunohistochemical staining for maspin in PDAC and adjacent normal pancreatic tissues. (A) Invasive PDAC has strong cytoplasmic maspin staining in the tumor cells (x400). (B) The normal pancreatic tissue adjacent to PDAC has no maspin-stained acinar or ductal cells (x400).

In addition, part of each PDAC tissue sample was frozen in liquid nitrogen immediately after surgical removal and maintained at $-70^{\circ} \mathrm{C}$. As a control reference, normal pancreatic tissues were obtained from different patients who had undergone resection surgery for a pancreatic endocrine tumor. In addition, as a control reference, normal breast tissues were obtained from different patients who had undergone resection surgery for benign breast disease. These tissues were frozen in liquid nitrogen using the method described above for the PDCA specimens. The clinical data were gathered by a medical records review. This study was approved by the institutional review board of Samsung Medical Center.

Immunohistochemistry. To investigate the expression of maspin in PDAC and adjacent normal pancreatic tissue, $4 \mu \mathrm{m}$ sections were obtained from the paraffin-embedded PDAC blocks. Immunoperoxidase staining was performed using purified primary antibodies against maspin (Pharmingen, San Diego, CA, 1:3,000). Negative controls were obtained by omitting the primary antibody, while normal breast tissue served as the positive control.

Cells with either granular cytoplasmic/nuclear or diffuse cytoplasmic staining were scored as positive for maspin expression. The quick scores were calculated using estimates for both the proportion and intensity of the stained cells. The proportion was scored as 0 ( 0 positive cells out of 100 tumor cells), $1(<1 / 100$ of the tumor cells are maspin-positive), 2 (1/100 to $1 / 10$ of the tumor cells), 3 (1/10 to $1 / 3$ of the tumor cells), 4 ( $1 / 3$ to $2 / 3$ of the tumor cells), or 5 ( $>2 / 3$ of the tumor cells), while the intensity was measured as $0,1,2$ or 3 , which corresponded to no staining, weak, moderate, or strong staining, respectively.

Genotyping. Genomic DNA was extracted from the formalinfixed, paraffin-embedded PDAC blocks by microdissection. After cells were selectively procured, DNA was isolated and then purified by phenol-chloroform extraction and alcohol precipitation. The DNA was PCR amplified using the following primers to detect $c .1022 \mathrm{G}>\mathrm{A}$ in the maspin gene: 5'-ATAACTGTGACTCCAGGCCC-3' (forward) and 5'-GGA CTTAACATGGGCTATGC-3' (reverse). The PCR products were sequenced using an ABI 3700 Automated Sequencer system (Applied Biosystems, Foster city, CA) at Macrogene Company (Seoul, Korea).

Human pancreatic cancer cell lines. The human PDACderived cell line Panc-1 was obtained from the Korean Cell Line Bank (KCLB ${ }^{\circledR}$, Seoul, Korea). This cell line was cultured on plastic plates in Dulbecco's modified Eagle's medium (DMEM), supplemented with $10 \%$ fetal calf serum, $1 \mathrm{mM}$ $\mathrm{NaHCO}_{3}$, and $2 \mathrm{mM}$ of glutamine and penicillin-streptomycin at $37^{\circ} \mathrm{C}$ in a $5 \% \mathrm{CO}_{2}$ incubator.

Western blotting. Both the cells derived from the fresh frozen tissue specimens and the Panc- 1 cell lines were lysed in RIPA buffer. Soluble protein from each extract $(30 \mu \mathrm{g})$ was run by $10 \%$ SDS-PAGE and then transferred to a nitrocellulose membrane. The membranes were incubated overnight with anti-maspin antibody (Pharmingen) at $4^{\circ} \mathrm{C}$. The membranes were then incubated with an HRP-conjugated secondary antibody for $\sim 30 \mathrm{~min}$ at room temperature, followed by detection using an enhanced chemiluminescence system (Amershan Corp., Arlington Heights, IL).

Maspin cDNA preparation and RT-PCR assay. Total RNA was isolated from the fresh frozen tissue specimens and the Panc-1 cell lines using TRIzol reagent (Invitrogen, Carsbad, CA) according to the manufacturer's recommendations. cDNA containing the entire maspin protein coding sequence was obtained by RT-PCR. The primers used were based on the nucleotide sequence for human maspin (GenBank accession no. U04313): 5'-AGACATTCTCGCTTCCCTGA-3' (forward) and 5'-AATTTTGACCCCTTATGGGC-3' (reverse). Ten microliters of cDNA, corresponding to $2 \mathrm{mg}$ of total RNA, was amplified in $20 \mathrm{ml}$ of a PCR reaction mixture containing 10X PCR buffer, $400 \mathrm{mM}$ each of dATP, dGTP, dCTP and dTTP, $0.2 \mathrm{mM}$ of each primer and 1.5 units of AmpliTaq DNA polymerase (Roche Diagnostics Co., Mannheim, Germany). The reactions were carried out for 35 cycles of $94^{\circ} \mathrm{C}$ for $60 \mathrm{sec}, 60^{\circ} \mathrm{C}$ for $190 \mathrm{sec}$, and $72^{\circ} \mathrm{C}$ for $60 \mathrm{sec}$, in a Peltier thermal cycler 200 (MJ Research, Inc. Waltham, MA). For the RT-PCR assay, the amplified products were analyzed by electrophoresis on a $1 \%$ agarose gel and then stained with ethidium bromide. 




(C)

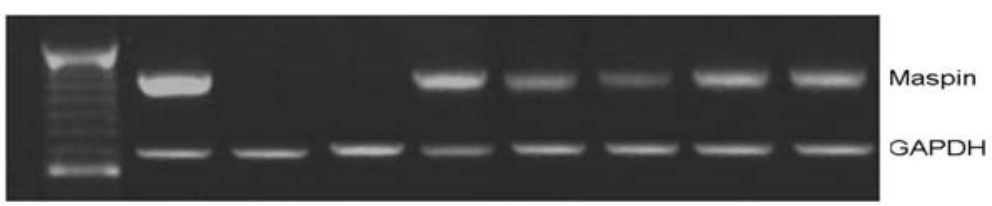

Figure 2. The expression of maspin in human PDAC tissues. Maspin protein and mRNA expression in fresh frozen normal pancreatic tissue (lane 3) and human PDAC tissue specimens (lane 4-8) were assessed by Western blotting, Northern blotting and RT-PCR. Normal breast tissue specimens were used as a positive control (lane 1), and human pancreatic cancer cell line Panc-1 was used as a negative control (lane 2). (A) Western blot analysis. Maspin expression was detectable in the PDAC tissues, although it was not detected in the normal pancreatic tissue. (B) Northern blot analysis. Enhanced maspin mRNA expression was seen in the PDAC tissues, whereas no maspin mRNA expression was seen in the normal pancreatic tissue. Ethidium bromide (EtBr) staining was used to label the total RNA. (C) RT-PCR. The $2.5 \mathrm{~kb}$ transcripts were expressed in PDAC tissues, but they were absent from the normal pancreatic tissue.

Northern blotting. For Northern blot hybridization, total RNA $(10 \mu \mathrm{g})$ was denatured in the presence of $50 \%$ formamide, 2.2 M formaldehyde, $20 \mathrm{mM}$ MOPS, $4 \mathrm{mM}$ sodium acetate and $0.5 \mathrm{mM}$ EDTA at $65^{\circ} \mathrm{C}$ for $10 \mathrm{~min}$. After electrophoresis in a $1.2 \%$ agarose gel containing $2.2 \mathrm{M}$ formaldehyde, RNA was vacuum blotted onto nylon membranes (Hybond-N+; Amersham Pharmacia biotech, Buckinghamshire, UK), and the filters were UV cross-linked. The cloned cDNA was used as a probe. The cDNA inserts were labeled with [ $\left.{ }^{32} \mathrm{P}\right]$-dATP (Amersham) using hexamer primers. Prehybridization, hybridization, washing, and autoradiography were performed as previously described in detail (16).

Construction of maspin-expressing stable transfectants. Maspin cDNA fragments were subcloned into pEGFPC1 (ClonTech, Palo Alto, CA) to yield the expression plasmid pEGFPC1-maspin. The maspin cDNA inserts were verified by nucleotide sequencing. Based on the $\mathrm{c} .1022 \mathrm{G}>\mathrm{A}$ variant, we considered the isoleucine (ATC) residue the wild-type and the valine (GTC) residue the mutant.

The pEGFPC1-maspin plasmid was transfected into Panc-1 cells using Effectene (Qiagen) according to the manufacturer's recommendations. As a control, the pEGFPC1 vector without a maspin cDNA insert was transfected into Panc- 1 cells. After 2 weeks of G418 selection, both the maspin-expressing clones (Panc-1-maspin) and mock-transfected clones (Panc-1-mock) were isolated. The stable transfectants were screened using a fluorescence microscope, and the conditioned DMEM media was subjected to Western blotting to assess maspin expression.

In vitro invasion assay. A rapid in vitro invasion assay was performed using Transwell chambers with polycarbonate membrane filters that had 8-mm diameter pores (Becton
Dickinson Labware, Bedford, MA) (17). To perform the invasion assay, a basement membrane complex (Matrigel; Becton Dickinson Labware) that contained laminin, collagen IV, and proteoglycan as the major components was applied to the membrane filter of the upper chamber $(10 \mu \mathrm{g} /$ filter $)$, and the filters were dried under a laminar flow hood. Subsequently, the filters were exposed to $500 \mu 1$ of warm culture medium, and they were rehydrated at $37^{\circ} \mathrm{C}$ for $1.5 \mathrm{~h}$. The lower chamber was filled with $1 \mathrm{ml}$ of conditioned medium. Cell suspensions of the Panc-1-maspin and the Pacn-1-mock clones were adjusted to a concentration of $10^{6}$ cells $/ \mathrm{ml}$ with $0.1 \% \mathrm{BSA}$, and $500 \mu \mathrm{l}$ of the adjusted cell suspension $\left(5 \times 10^{5}\right.$ cells/filter $)$ was immediately placed in the Matrigeltreated upper chamber. After incubation at $37^{\circ} \mathrm{C}$ for $24 \mathrm{~h}$ in a $5 \% \mathrm{CO}_{2}$ incubator, the cells on the upper surface of the filter were completely removed with cotton swabs. The filter was then stained with hematoxylin, and the invasive potential of the cells was determined by counting the number of cells that had migrated to the lower surface of the filter in 10 different areas. Each assay was performed in triplicate in 3 separate experiments.

Statistics. All statistical evaluations were carried out using a unpaired Student's t-test for continuous variables and $\chi^{2}$ test or Fisher's exact test for categorical variables. Statistical significance was tested at a probability level of 0.05 .

\section{Results}

Maspin expression in human PDAC tissues. Maspin expression in the PDAC and normal pancreatic tissues was assessed by immunohistochemistry on the paraffin-embedded PDAC and adjacent normal pancreatic tissues (Fig. 1). Expression was observed in all the PDAC tissues (40/40; 
Table I. The relationship between the c.1022G $>$ A variant genotype and the PDAC characteristics.

\begin{tabular}{llcccc}
\hline & & \multicolumn{3}{c}{ Genotype (n) } & P-value \\
\cline { 3 - 4 } & Clinical characteristics & AA & AG & GG & \\
\hline No. & & 18 & 7 & 15 & 0.306 \\
Age & $(<60 / \geq 60$ years $)$ & $10 / 8$ & $4 / 3$ & $12 / 3$ & 0.225 \\
Gender & (Male/female) & $11 / 7$ & $5 / 2$ & $10 / 5$ & 0.989 \\
Maspin & (Quick score 5/6/7/8) & $1 / 6 / 7 / 4$ & $0 / 2 / 3 / 2$ & $1 / 5 / 4 / 5$ & 0.757 \\
Tumor stage & (I/II/III/IV) & $5 / 3 / 5 / 5$ & $2 / 0 / 3 / 2$ & $3 / 0 / 8 / 4$ & 0.911 \\
Lymph node & (Negative/positive) & $9 / 9$ & $4 / 3$ & $6 / 9$ & 0.244 \\
Tumor location & (Head/body or tail) & $16 / 2$ & $5 / 2$ & $13 / 2$ & 0.822 \\
Differentiation & (Well/moderate/poor) & $4 / 12 / 2$ & $0 / 5 / 2$ & $6 / 8 / 1$ & 0.906 \\
CA 19-9 & $(<37 / \geq 37$ IU/ml) & $4 / 14$ & $4 / 3$ & $5 / 10$ & \\
\hline
\end{tabular}

(A)

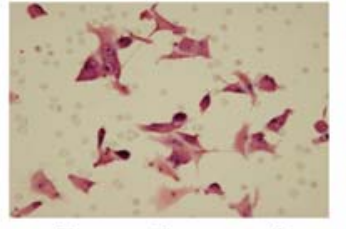

Panc-1-maspin

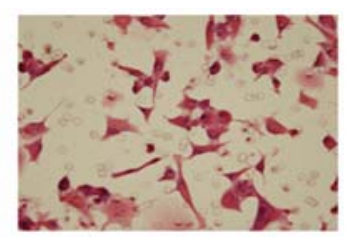

Panc-1-mock
(B)

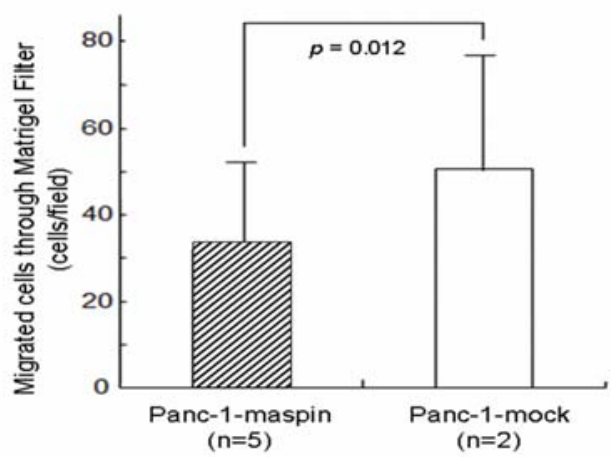

Figure 3. In vitro invasion assay. (A) Photomicrographs (x400) showing Panc-1-maspin (left) and Panc-1-mock (right) cells that migrated to the lower surface of the Matrigel-coated filter. The cells were plated on the upper surface of these filters and incubated for $24 \mathrm{~h}$ before staining with hematoxylin. (B) The invasive ability of the maspin-expressing Panc-1maspin cells was significantly suppressed compared with that of the Panc-1mock cells in the in vitro invasion assay $(\mathrm{p}=0.012)$.

100\%). Maspin immunoreactivity was mainly cytoplasmic, but there was some nuclear staining. All the PDAC tissues had quick scores between 5 and 8 . Unlike the PDAC tissues, the corresponding normal pancreatic tissues showed little or no maspin expression.

Maspin protein and RNA expression in the fresh frozen normal pancreatic and PDAC tissue specimens were assessed by Western blotting, RT-PCR assay, and Northern blotting (Fig. 2). The normal breast tissue specimens were used as a positive control, and the human pancreatic cancer cell line Panc-1 was used as a negative control. The Panc- 1 cell line is known to be highly invasive, but it lacks maspin expression

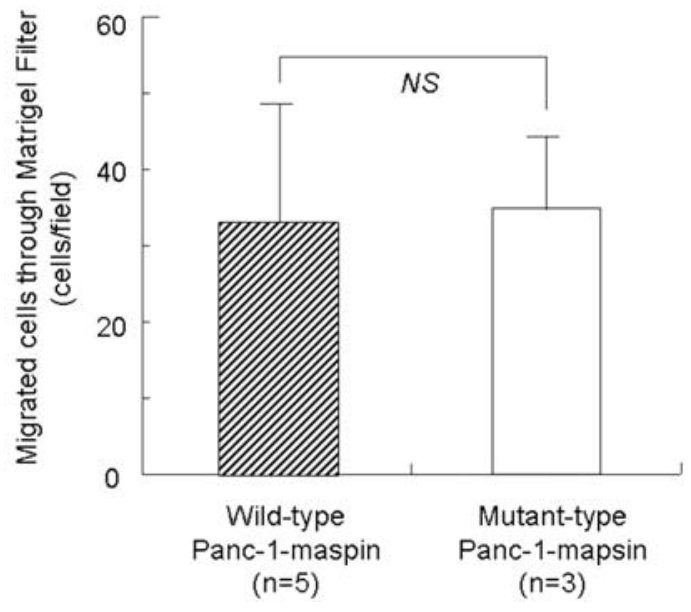

Figure 4. Comparison of the invasive ability of wild-type Panc-1-maspin cells and mutant Panc-1-maspin cells.

$(10,12,17-19)$. Both the maspin protein and RNA were abundantly expressed in the PDAC specimens as assayed by Western and Northern blotting, whereas expression was not detected in the normal pancreatic tissue specimens. In addition, the $2.5 \mathrm{~kb}$ transcripts were detected by RT-PCR in PDAC tissues, but they were not expressed in normal pancreatic tissue.

The effect of wild-type maspin on the invasive ability of PDAC cells. Maspin cDNA was obtained from the fresh frozen PDAC specimen by performing RT-PCR and was subcloned into pEGFPC1. After transfecting pEGFPC1maspin into Panc-1, which is a PDAC-derived cell line that lacks maspin expression, we isolated five independent G418resistant Panc-1-maspin clones.

The invasive ability of the Panc-1-maspin and Panc-1mock cells was evaluated with an in vitro invasion assay. No change in cell shape was detected in the transfected cells (Fig. 3A). The baseline invasion ability of the parental Panc-1 cells ( $44.2 \pm 24.5$ cells/field) showed no significant difference from that of the Panc-1-mock cells. In the in vitro invasion assay, the average numbers of cells from two 
independent Panc-1-mock clones that migrated through a Matrigel-coated filter were $42.7 \pm 21.1$ cells/field and $58.3 \pm 32.6$ cells/field. The average numbers of cells from five independent Panc-1-maspin clones that migrated through a Matrigel-coated filter were $29.4 \pm 21.2$ cells/field, $34.0 \pm 23.1$ cells/field, $24.1 \pm 17.6$ cells/field, $29.4 \pm 18.1$ cells/field, and $43.4 \pm 22.2$ cells/field. Thus, the ability of Panc-1-maspin cells to migrate through a Matrigel-coated filter was significantly suppressed compared with that of the Panc-1-mock cells in a $24 \mathrm{~h}$ in vitro invasion assay ( $\mathrm{p}=0.012$, Fig. 3B). This result indicates that wild-type maspin suppresses the invasive ability of PDAC cells.

Genotyping the maspin gene. Through direct sequencing of the maspin genomic DNA isolated from human PDAC tissues, c.1022G $>$ A was identified (Table I). While the AA genotype was most prevalent (18 of the 40 samples), the GG genotype was found in 15 of the 40 samples, and the AG genotype was revealed in 7 of the 40 samples. These results indicated that the c. $1022 \mathrm{G}>\mathrm{A}$ variant was present almost as frequently as the wild-type A variant.

We also assessed the relationship between each maspin genotype and the PDAC phenotypes. To evaluate the effect of the maspin c.1022A $>\mathrm{G}$ variant on the invasive ability of PDAC cells, we constructed two stable transfectants that expressed mutant maspin cDNA and performed an in vitro invasion assay. The average number of cells of the mutant Panc-1-maspin clones that migrated through a Matrigelcoated filter was $33.6 \pm 17.2$ cells/field. Thus, there was no difference in invasive ability between the wild-type and mutant Panc-1-maspin cells (Fig. 4).

\section{Discussion}

Maspin was proposed to be a tumor suppressor based on its differential expression in normal mammary epithelial cells and breast carcinoma cell lines (1). Interestingly, maspin expression in human PDAC tissue has been shown to be paradoxical; while low or no maspin expression was observed in the normal pancreatic tissues, maspin overexpression was observed in most human PDACs. Unlike previous studies that only immunohistochemically stained human PDAC tissue (10-13), we examined maspin protein and RNA expression levels in fresh frozen human PDAC and normal pancreatic tissue specimens by performing immunohistochemistry, Western blotting, RT-PCR, and Northern blotting. Our results reconfirmed that maspin protein and RNA were strongly expressed in human PDAC tissues and that they were weakly expressed or not expressed at all in normal pancreatic tissues. Several microarray analyses have revealed that the expression of maspin is tissue-specific and that it frequently has higher expression in tumor tissues than in normal tissues (20). It has been established that tissuespecific expression of maspin is regulated by epigenetic changes that occur in the maspin promoter region, including Ets, activator protein 1, hormone-responsive element, HIF and p53 binding sites $(2,18,19,21,22)$. These changes involve cytosine methylation and histone deacetylation.

Interestingly, the expression pattern and prognostic significance of maspin in ovarian cancer appears to be very similar to that of PDAC (23). In experiments on maspin in ovarian cancer, introducing wild-type maspin into ovarian cancer cells reduced their invasiveness (23). However, there have been no previous studies about the function of aberrantly expressed maspin or its effect on invasion and metastasis in PDAC. In this study, we found that aberrantly expressed maspin in human PDAC could also suppress the invasive ability of PDAC cells. Generally, while the majority of $\mathrm{CpG}$ islands of somatic tissues are methylated, tumor cells have global DNA hypomethylation compared with their normal counterparts (24). Hypomethylation is involved in the progression from the premalignant state to a fully developed malignancy (25). Therefore, it is possible that inducing maspin expression through the loss of promoter methylation is simply the result of a non-specific event associated with the genome-wide epigenetic changes that occur in the genome of cancer cells (19). This possibility could explain why maspin expression in PDAC is aberrant and also why aberrantly expressed maspin has a tumor suppressive function as it does in breast tissues. However, because aberrantly expressed maspin in PDAC was associated with a poor prognosis, further study is needed to confirm the role of aberrantly expressed maspin. Aberrantly expressed maspin may have different functions depending on the spectrum of other interacting proteins that are expressed in vivo.

In addition, we identified the maspin c. $1022 \mathrm{~A}>\mathrm{G}$ variant in PDAC and evaluated its significance. Although this variant results in a substitution of valine for isoleucine, there was no causative link between the allelic variant and the clinical characteristics of PDAC or the altered invasive ability of the PDAC cells.

The results of the present study indicate that maspin suppresses the invasive ability of PDAC cells, but the maspin c. $1022 \mathrm{~A}>\mathrm{G}$ variant was not associated with any tumor phenotypes or functional alteration of maspin in PDAC. To precisely determine the cellular and biochemical activities of maspin in PDAC, further studies on the divergent mechanisms of maspin are necessary.

\section{References}

1. Zou Z, Anisowicz A, Hendrix MJ, Thor A, Neveu M, Sheng S, Rafidi K, Seftor E and Sager R: Maspin, a serpin with tumorsuppressing activity in human mammary epithelial cells. Science 263: 526-529, 1994.

2. Maass N, Biallek M, Rosel F, Schem C, Ohike N, Zhang M, Jonat W and Nagasaki K: Hypermethylation and histone deacetylation lead to silencing of the maspin gene in human breast cancer. Biochem Biophys Res Commun 297: 125-128, 2002.

3. Sheng S, Carey J, Seftor EA, Dias L, Hendrix MJ and Sager R: Maspin acts at the cell membrane to inhibit invasion and motility of mammary and prostatic cancer cells. Proc Natl Acad Sci USA 93: 11669-11674, 1996.

4. Zhang M, Volpert O, Shi YH and Bouck N: Maspin is an angiogenesis inhibitor. Nat Med 6: 196-199, 2000.

5. Jiang N, Meng Y, Zhang S, Mensah-Osman E and Sheng S: Maspin sensitizes breast carcinoma cells to induced apoptosis. Oncogene 21: 4089-4098, 2002.

6. Bailey CM, Khalkhali-Ellis Z, Seftor EA and Hendrix MJ: Biological functions of maspin. J Cell Physiol 209: 617-624, 2006.

7. Futscher BW, O'Meara MM, Kim CJ, Rennels MA, Lu D, Gruman LM, Seftor RE, Hendrix MJ and Domann FE: Aberrant methylation of the maspin promoter is an early event in human breast cancer. Neoplasia 6: 380-389, 2006. 
8. Seftor RE, Seftor EA, Sheng S, Pemberton PA, Sager R and Hendrix MJ: maspin suppresses the invasive phenotype of human breast carcinoma. Cancer Res 58: 5681-5685, 1998.

9. Shi HY, Zhang W, Liang R, Abraham S, Kittrell FS, Medina D and Zhang M: Blocking tumor growth, invasion, and metastasis by maspin in a syngeneic breast cancer model. Cancer Res 61 : 6945-6951, 2001

10. Maass N, Hojo T, Ueding M, Luttges J, Kloppel G, Jonat W and Nagasaki K: Expression of the tumor suppressor gene Maspin in human pancreatic cancers. Clin Cancer Res 7: 812-817, 2001.

11. Oh YL, Song SY and Ahn G: Expression of maspin in pancreatic neoplasms: application of maspin immunohistochemistry to the differential diagnosis. Appl Immunohistochem Mol Morphol 10: 62-66, 2002.

12. Ohike N, Maass N, Mundhenke C, Biallek M, Zhang M, Jonat W, Luttges J, Morohoshi T, Kloppel G and Nagasaki K: Clinicopathological significance and molecular regulation of maspin expression in ductal adenocarcinoma of the pancreas Cancer Lett 199: 193-200, 2003.

13. Lim YJ, Lee JK, Jang WY, Song SY, Lee KT, Paik SW and Rhee JC: Prognostic significance of maspin in pancreatic ductal adenocarcinoma. Korean J Intern Med 19: 15-18, 2004

14. Cao D, Zhang Q, Wu LS, Salaria SN, Winter JW, Hruban RH, Goggins MS, Abbruzzese JL, Maitra A and Ho L: Prognostic significance of maspin in pancreatic ductal adenocarcinoma: tissue microarray analysis of 223 surgically resected cases. Mod Pathol 20: 570-578, 2007.

15. Umekita Y, Hiipakka RA and Liao S: Rat and human maspins: structures, metastatic suppressor activity and mutation in prostate cancer cells. Cancer Lett 113: 87-93, 1997.

16. Lee SH, Zhang W, Choi JJ, Cho YS, Oh SH, Kim JW, Hu L, $\mathrm{Xu}$ J, Liu J and Lee JH: Overexpression of the thymosin beta-10 gene in human ovarian cancer cells disrupts F-actin stress fiber and leads to apoptosis. Oncogene 20: 6700-6706, 2001.
17. Tajima H, Ohta T, Elnemr A, Yasui T, Kitagawa H, Fushida S, Kayahara M, Miwa K, Wakayama T, Iseki S and Yokoyama S: Enhanced invasiveness of pancreatic adenocarcinoma cells stably transfected with cationic trypsinogen cDNA. Int J Cancer 94: 699-704, 2001.

18. Fitzgerald M, Oshiro M, Holtan N, Krager K, Cullen JJ, Futscher BW and Domann FE: Human pancreatic carcinoma cells activate maspin expression through loss of epigenetic control. Neoplasia 5: 427-436, 2003.

19. Sato N, Fukushima N, Matsubayashi H and Goggins M: Identification of maspin and S100P as novel hypomethylation targets in pancreatic cancer using global gene expression profiling. Oncogene 23: 1531-1538, 2004.

20. Khalkhali-Ellis Z: Maspin: the new frontier. Clin Cancer Res 12: 7279-7283, 2006

21. Domann FE, Rice JC, Hendrix MJ and Futscher BW: Epigenetic silencing of maspin gene expression in human breast cancers. Int J Cancer 85: 805-810, 2000.

22. Rose SL, Fitzgerald MP, White NO, Hitchler MJ, Futscher BW, De Geest K and Domann FE: Epigenetic regulation of maspin expression in human ovarian carcinoma cells. Gynecol Oncol 102: 319-324, 2006.

23. Sood AK, Fletcher MS, Gruman LM, Coffin JE, Jabbari S, Khalkhali-Ellis Z, Arbour N, Seftor EA and Hendrix MJ: The paradoxical expression of maspin in ovarian carcinoma. Clin Cancer Res 8: 2924-3932, 2002.

24. Feinberg AP and Vogelstein B: Hypomethylation distinguishes genes of some human cancers from their normal counterparts. Nature 301: 89-92, 1983.

25. Dunn BK: Hypomethylation: one side of a larger picture. Ann NY Acad Sci 983: 28-42, 2003. 